\title{
Adsorption of 1-Decyl-3-methylimidazolium Bromide and Solvation Structure of Bromide at the Air/Water Interface
}

\author{
Makoto Aratono, ${ }^{* 1 \dagger}$ Kei Shimamoto, ${ }^{* 1}$ Asuka Onohara, ${ }^{* 1}$ Daiki Murakami, ${ }^{* 1}$ Hajime Tanida, ${ }^{* 2}$ \\ Iwao Watanabe, ${ }^{* 3}$ Toru OzEKI, ${ }^{* 4}$ Hiroki Matsubara, ${ }^{* 1}$ and Takanori TAKIUE*1 \\ *1 Department of Chemistry, Faculty of Sciences, Kyushu University, Fukuoka 812-8581, Japan \\ *2 Experimental Facilities Division, Japan Synchrotron Radiation Research Institute, Hyogo 679-5198, Japan \\ *3 Department of Chemistry, Graduate School of Science, Osaka Prefecture University, \\ Sakai, Osaka 599-8531, Japan \\ *4 Department of Natural Sciences, Hyogo University of Teacher Education, Hyogo 673-1494, Japan
}

\begin{abstract}
The adsorbed film of 1-decyl-3-methylimidazolium bromide (DeMIMB) at the air/water interface was investigated employing the surface tension measurement and the x-ray absorption fine structure method under the total reflection condition (TRXAFS). From the surface tension measurement, the surface excess concentrations of ions were determined. From the XAFS measurement, two solvation states of bromide ion were found in the adsorbed film, which were assigned to be "free-Br" and "bound-Br". The hydration number of the former was estimated to be 6 while that of the latter was estimated to be 4 . The results based on the XAFS analysis provided significant information on the formation of domains in the adsorbed film; the most conceivable situation is that the adsorbed molecules are definitely not homogeneously dispersed, but domains (islands or clusters) are dispersed in the adsorbed film. A regular and rather tight stacking of immidazolium rings may be formed in the domains.
\end{abstract}

(Received June 4, 2008; Accepted July 7, 2008; Published October 10, 2008)

\section{Introduction}

Melting points of organic electrolytes are usually lower compared to inorganic electrolytes and liquid state is often realized even at room temperature by employing suitable combination of cationic and anionic ions. Such electrolytes with a low melting point are referred to as ionic liquids (IL). Several kinds of synthesized dialkylimidazolium ionic liquids are stable in the atmospheric environment ${ }^{1,2}$ and thus have attracted much attention as novel alternative solvents of the common organic solvents, ${ }^{3-5}$ materials for electrochemical cells ${ }^{6,7}$ triborogical materials ${ }^{8,9}$ and so on. Since widespread physical and chemical properties of ILs are essential not only for fully understanding why ILs are liquid even at low temperatures but also for proper choice of ILs for a specific application, such data have been accumulating extensively. ${ }^{10-14}$

Although the most important aspect of ILs is that they are liquid even at low temperatures, another aspect is that ILs have basically amphiphilic chemical structure. From these points of view, a wide variety of colloid and interface science is expected by using ILs similarly to liquid polyethylene glycol surfactants. ${ }^{15}$ Actually many reports have been published with respect to surface properties and structures not only of neat ILs but also of their aqueous solutions. ${ }^{16-25}$ Nevertheless, researches on the surface properties and structures of IL systems are not satisfactory but rather have just started in these last few years. The present study was performed with a view to elucidating the

† To whom correspondence should be addressed.

E-mail: aratono@chem.kyushu-univ.jp adsorption of 1-decyl-3-methylimidazolium bromide (DeMIMB), a typical of IL, at the air/water surface from the viewpoints of the macroscopic thermodynamic quantity such as surface excess concentration and the molecular level structure such as the coordination number of bromide ions. For this purpose we employed the surface tension measurements and the $\mathrm{X}$-ray absorption fine structure method under the total reflection condition (TRXAFS).

\section{Experimental}

\section{Materials}

DeMIMB was synthesized by mixing 1-bromodecane (Aldrich, 99\%), which had been purified by distillation at around $370 \mathrm{~K}$ under reduced pressure of $3 \mathrm{mmHg}$, and 1-methylimidazole (Aldrich 99\%) in anhydrous toluene at the temperature of an ice-water bath. The crude product was recrystallized three times from toluene and ethylacetate. The purity was confirmed by absence of a minimum on the surface tension $v s$. concentration curve around the critical micelle concentration $(\mathrm{cmc})$. Water was first passed through an ion exchange apparatus (EYELA, SA2000A) and then distilled three times, where the second and third stages were done from alkaline permanganate solution to decompose and remove organic impurities.

\section{Surface tension}

The surface tension $\gamma$ of the aqueous solution was measured as a function of the molality of DeMIMB at 298.15 K under atmospheric pressure by means of the drop volume method, 


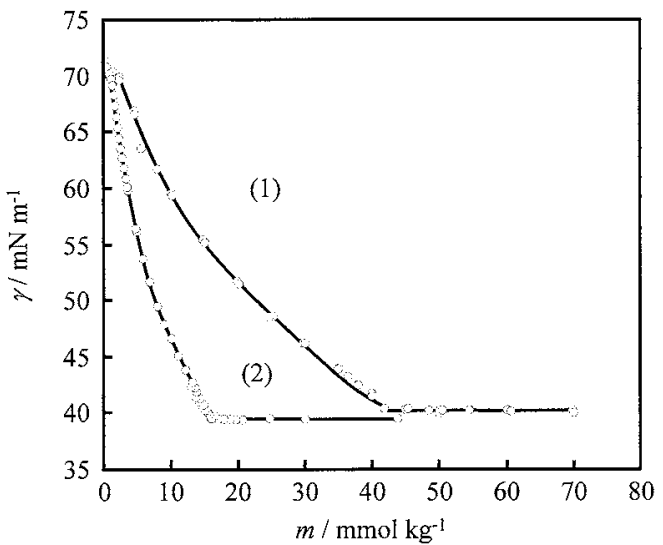

Fig. 1 Surface tension vs. molality curves. (1) DeMIMB, (2) DTAB.

where the volume of a drop $V$ is related to the surface tension via

$$
\gamma=(V \Delta \rho g / r) F
$$

where $\Delta \rho$ is the density difference between aqueous solution and air surrounding it, ${ }^{26} g$ the acceleration of gravity, $r$ the radius of the glass capillary tip, and $F$ the correction factor given as a function of $r / V^{1 / 3} .27$ The $\gamma$ values were reproducible within \pm 0.05 $\mathrm{mN} \mathrm{m}^{-1}$.

\section{Total reflection $X A F S$}

The XAFS experiments were performed at BL-7C of the Photon Factory of the National Laboratory for High Energy Accelerator Research Organization (Tsukuba, Japan). The XAFS method was applied to the air/water surface under the total reflection condition, which we call the TRXAFS method. The x-ray beam monochromatized by a double-crystal monochromator [Si-(111)] strikes the solution surface of a trough under the total reflection conditions at about $1 \mathrm{mrad}$. The incident beam intensity $I_{0}$ was measured by a gas ionization chamber filled with nitrogen, the signal intensity $I$ was detected by the total-conversion helium ion yield method, and then the $I / I_{0}$ vs. photon energy plots were obtained. The principle, procedures of experiments, and the schematic views of the method were fully described in our previous papers. ${ }^{28-31}$

\section{Results and Discussion}

The surface tension $\gamma$ of the aqueous solution is plotted against the molality $m$ of DeMIMB in Fig. 1 . The $\gamma$ value goes down with increasing $m$ and becomes almost constant above a break point. Since the aqueous solution was not turbid but transparent within the concentration range measured, it was concluded that DeMIMB molecules form normal micelles similarly to usual cationic surfactants like dodecyltrimethylammonium bromide (DTAB). The surface tension is compared with that of the DTAB solution in Fig. 1..$^{32,33}$

Surface states from a macroscopic viewpoint have been examined usually in terms of a surface excess concentration $\Gamma_{\mathrm{i}}^{\mathrm{H}}$ of adsorbed species $i$ and the surface pressure $\pi v s$. average area per species $A_{\mathrm{i}}$ curve. Here $\pi$ and $A$ are defined by

$$
\pi=\gamma^{0}-\gamma
$$

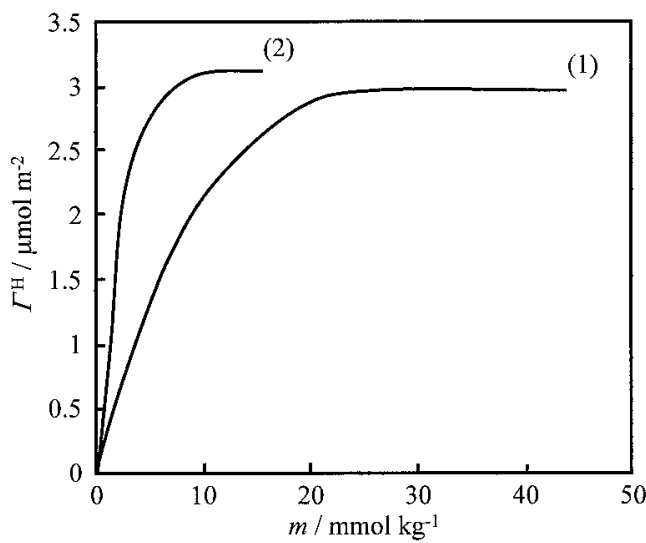

Fig. 2 Surface excess concentration vs. molality curves. DeMIMB, (2) DTAB

$$
A_{\mathrm{i}}=1 / N_{\mathrm{A}} \Gamma_{\mathrm{i}}^{\mathrm{H}}
$$

respectively, where $\gamma^{0}$ is the surface tension of pure water and $N_{\mathrm{A}}$ the Avogadro number. The total differential of $\gamma$ is written as $^{34}$

$$
\mathrm{d} \gamma=-\Delta s \mathrm{~d} T+\Delta v \mathrm{~d} p-\left(\Gamma_{+}{ }^{\mathrm{H}}+\Gamma_{-}{ }^{\mathrm{H}}\right)(R T / m) \mathrm{d} m
$$

where $\Delta s$ and $\Delta v$ are the entropy and volume changes associated with adsorption and the aqueous solution is assumed to be ideally dilute. Since the electroneutrality condition is required for $\Gamma_{\mathrm{i}}^{\mathrm{H}}$ introduced here, the relation

$$
\Gamma_{+}^{\mathrm{H}}=\Gamma_{-}^{\mathrm{H}} \equiv \Gamma^{\mathrm{H}}
$$

should hold. At constant temperature and pressure, thus we have

$$
\Gamma^{\mathrm{H}}=-(m / 2 R T)(\partial \gamma / \partial m)_{\mathrm{T}, \mathrm{p}}
$$

The surface excess concentration of DeMIM+ ${ }^{+}$ions calculated by applying Eq. (6) to the curves in Fig. 1 is shown in Fig. 2 . It increases with $m$ up to a maximum value of $\Gamma^{\mathrm{H}}$ (around 2.9 $\mu \mathrm{mol} \mathrm{m}{ }^{-2}$ ) corresponding to the area $A$ of about $0.57 \mathrm{~nm}^{2}$. Since the number of ion pairs is also equal to $\Gamma^{\mathrm{H}}$, the minimum of the average area per ion pair is also $0.57 \mathrm{~nm}^{2}$. Referring to the fact that the geometrical size of unsolvated DeMIM $^{+}$ion is at most $0.36 \mathrm{~nm}^{2}$ for a freely rotating head group and that of $\mathrm{Br}^{-}$is 0.1 $\mathrm{nm}^{2}$, respectively as drawn in Fig. 3, a side by side arrangement of the ion pair at the surface is possible in principle even at the maximum adsorption. Such a side by side arrangement has been reported with respect to the adsorbed films of imidazorium ionic liquids having $\mathrm{BF}_{4}^{-}$as their counter ion on the basis of the results of the sum frequency generation method applied to the adsorbed film, ${ }^{19}$ the phase diagram of adsorption of an ionic liquid and alcohol mixture, ${ }^{22}$ and the surface properties of neat ionic liquids. ${ }^{23}$ Therefore, it is an important issue to get information on the location of $\mathrm{Br}^{-}$at the air/water interface and then on the difference of distributions at the interface among counter ion species. An examination of hydration state of $\mathrm{Br}$ using the XAFS method is expected to be advantageous on this important issue.

The characteristic advantages of the XAFS method are as follows: the spectrum around K-edge is ion-selective, its fine structure provides information on local structure around the selected element, and especially it is available even for liquid 


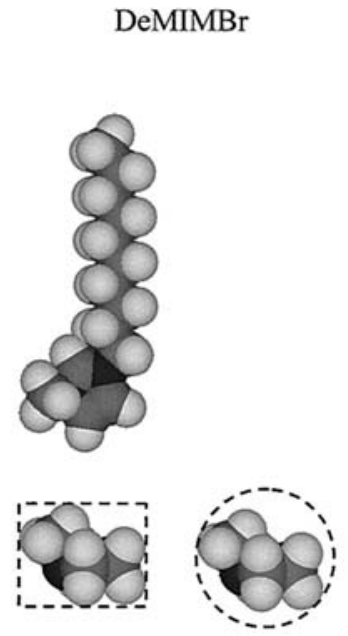

$0.23 \sim 0.36 \mathrm{~nm}^{2}$
DTAB
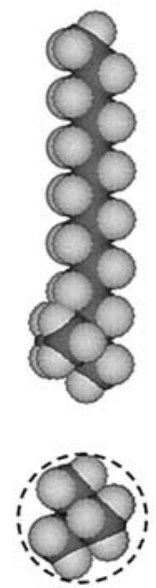

$0.35 \mathrm{~nm}^{2}$

Fig. 3 Chemical structures and estimated sizes of surfactant and counter ions.

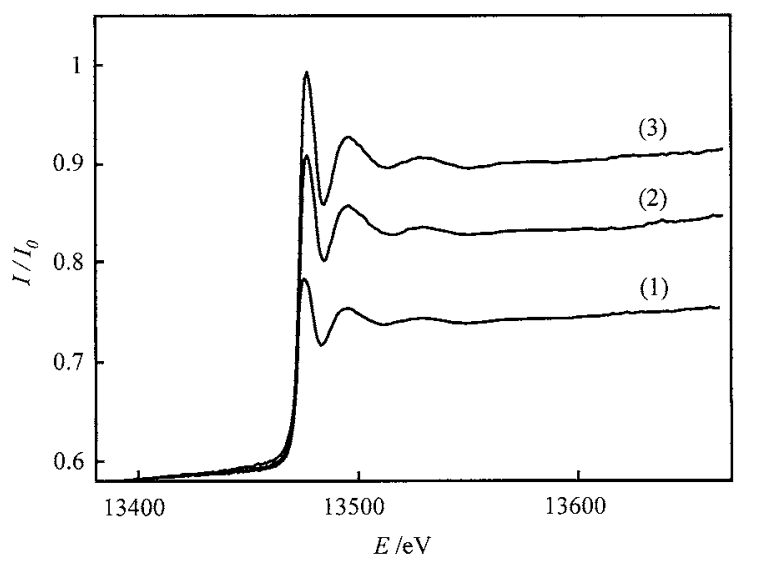

Fig. 4 K-edge $\mathrm{x}$-ray absorption spectra of bromide ions of DeMIMB at different molalities. $m / \mathrm{mmol} \mathrm{kg}^{-1}$ : (1) 6 , (2) 32 , (3) 50 .

and amorphous materials. We have applied the XAFS under the total reflection condition (TRXAFS) to the adsorbed films at the air/water interface and obtained not only the surface concentrations from the K-edge absorption intensity but also solvation structure of surface adsorbed ions from the XAFS analysis. ${ }^{28,35-38}$ Thus TRXAFS was applied to obtain the information on solvation structure of bromide ions in the adsorbed film of DeMIMB.

The signal intensity $v s$. photon energy $E$ curves are displayed at the representative three concentrations in Fig. 4, where the background absorption was subtracted. ${ }^{37}$ The jump around $13475 \mathrm{eV}$ is the K-edge absorption of bromide and increases with the DeMIMB bulk concentration, which corresponds to the increase in $\Gamma^{\mathrm{H}}$ shown in Fig. 2. To examine the solvation structure of bromide ions, the EXAFS analysis was applied to all the data obtained. The details of the EXAFS analysis will be fully demonstrated in a separate paper, ${ }^{37}$ and here the essentials are briefly described. First the $\chi$ spectra were calculated by

$$
k=\sqrt{2 m_{\mathrm{e}}\left(E-E_{0}\right) / \hbar^{2}}
$$

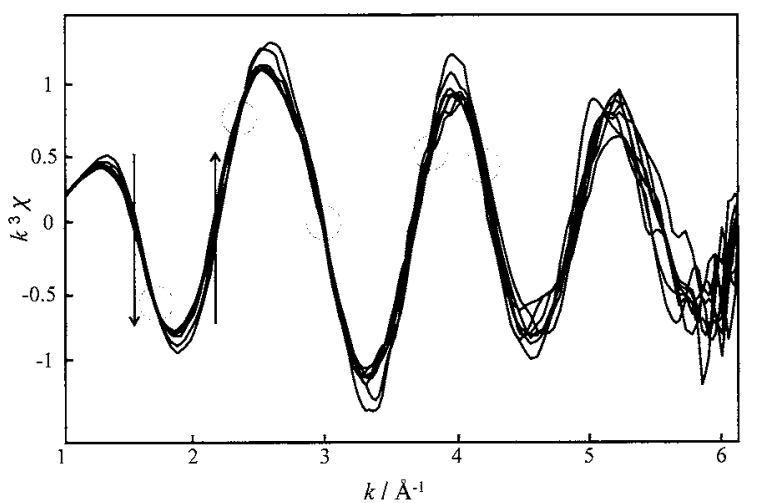

Fig. 5 K-edge EXAFS $k^{3} \chi$ spectra of bromide ions at different molalities. Arrows indicate the increase in molality.

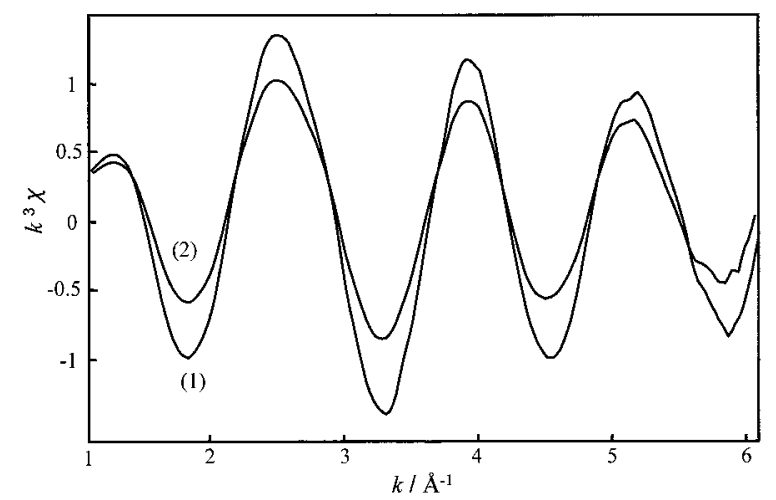

Fig. 6 Two spectra extracted from the K-edge EXAFS $k^{3} \chi$ spectra of bromide ions at different molalities. (1) Free-Br, (2) bound-Br.

and

$$
\chi(k)=\left[\mu(k)-\mu_{0}(k)\right] / \mu_{0}(k)
$$

where $k$ is the photoelectron wave vector, $m_{\mathrm{e}}$ the electron mass, $E$ the incident x-ray energy, $E_{0}$ the threshold energy for the Kedge absorption, $\mu(k)$ the experimental absorption coefficient due to the K-shell excitation, and $\mu_{0}(k)$ that of a hypothetical isolated bromide atom. The $k^{3} \chi$ spectra thus calculated are demonstrated at all the concentrations measured in Fig. 5.

The isosbestic points clearly exist and thus each $\chi$ spectrum is considered to be the sum of two specific $\chi$ spectra. The factor analysis performed on these spectral data should provide us with the two specific component spectra, $\chi_{1}$ and $\chi_{2}$, and their fractions $a_{1}$ and $a_{2}$, respectively, thus the $\chi$ spectrum at a given molality, $\chi_{\mathrm{m}}$, can be expressed as

$$
\chi_{\mathrm{m}}=a_{1, \mathrm{~m}} \chi_{1}+a_{2, \mathrm{~m}} \chi_{2}
$$

where $a_{1, \mathrm{~m}}$ and $a_{2, \mathrm{~m}}$ are the fractions $a_{1}$ and $a_{2}$ at the given molality $m\left(\mathrm{mmol} \mathrm{kg}^{-1}\right)$. Furthermore the detailed analysis showed that $a_{1,8}=0.95$ and $a_{1,50}=0.4$ could trace most satisfactorily all the $k^{3} \chi_{\mathrm{m}}$ spectra. ${ }^{37}$ The $\chi_{1}$ and $\chi_{2}$ spectra thus determined are demonstrated in Fig. 6. The next stage is to extract structure parameters from the $\chi_{1}$ and $\chi_{2}$ spectra by applying the equation 37,39 
Table 1 EXAFS curve-fitting results

\begin{tabular}{lcccccc}
\hline & Shell & $N$ & $r / \AA$ & $\sigma / \AA$ & $\Delta E_{0} / \mathrm{eV}$ & $R_{\mathrm{f}}, \%$ \\
\hline Free & 1 & 6 & 3.2 & 0.197 & -1.41 & 5.31 \\
Bound & 1 & 4 & 3.2 & 0.189 & -1.87 & 4.18 \\
& 2 & 3 & 4.2 & 0.386 & 9.35 & \\
\hline
\end{tabular}

$$
\begin{gathered}
\chi_{\mathrm{j}}=\left[B_{\mathrm{j}} F_{\mathrm{j}}\left(k_{\mathrm{j}}\right) / k_{\mathrm{j}} r_{\mathrm{j}}{ }^{2}\right] \exp \left[-2 r_{\mathrm{j}} / \lambda\right] \exp \left[-2 \sigma_{\mathrm{j}}{ }^{2} k_{\mathrm{j}}{ }^{2}\right] \times \\
\sin \left[2 k_{\mathrm{j}} r_{\mathrm{j}}+\phi_{\mathrm{j}}\left(k_{\mathrm{j}}\right)-k_{\mathrm{j}}{ }^{3} C_{3 \mathrm{j}}\right]
\end{gathered}
$$

where

$$
k_{\mathrm{j}}=\left[k^{2}-\left(2 m_{\mathrm{e}} / \hbar^{2}\right) \Delta E_{0 \mathrm{j}}\right]^{1 / 2}
$$

Here the subscript $j$ is the coordination shell number and $\lambda$ is the photoelectron mean free path. The parameters determined by fitting the experimental $k^{3} \chi$ spectra with Eq. (10) are the distance $r$ between absorbing and scattering atoms, the DebyeWaller like factor $\sigma$, the amplitude factor $B$, and the absorption edge shift $\Delta E_{0}$. The back scattering amplitude of photoelectron $F$ and the total phase shift $\phi$ were generated by the computer program FEFF ver. $8.10^{40}$ for the distance between $\mathrm{Br}$ and $\mathrm{O}$ atom $r_{\mathrm{Br}-\mathrm{O}}=3.2 \AA$. The anhamononicity $C_{3}$ was fixed at $34.4 \times$ $10^{-4} \AA .^{41}$

Since the spectrum $\chi_{1}$ was similar in shape to that of aqueous $\mathrm{NaBr}$ solution at low concentrations, the number of scattering atoms was assumed to be six. ${ }^{42}$ The distance between the scattering atoms and $\mathrm{Br}^{-}$thus determined was about $3.2 \AA$, which is close to the distance estimated by neutron diffraction experiments of the aqueous $\mathrm{KBr}$ solution. ${ }^{43}$ On the other hand, the analysis of the $\chi_{2}$ spectrum manifested that there were about four scattering atoms at around $3.2 \AA$ in the first shell and about three atoms at around $4 \AA$ in the second shell, respectively. Comparing with the results of the $\chi_{1}$ spectrum, it is reasonably said that the hydration number of $\chi_{2}$ state is about four. Let us call the states of $\mathrm{Br}^{-}$of $\chi_{1}$ and $\chi_{2}$ "free-Br" and "bound-Br", respectively. The results of analysis are summarized in Table 1. The fraction $a_{1, \mathrm{~m}}$ of "free-Br" goes down and $a_{2, \mathrm{~m}}$ of "bound-Br" goes up gradually with increasing the surface excess concentration $\Gamma^{\mathrm{H}}$ and at the $\mathrm{cmc}\left(\Gamma^{\mathrm{H}}=2.9 \mu \mathrm{mol} \mathrm{m}{ }^{-2}\right)$ those values for "free-Br" and "bound-Br" are 0.4 and 0.6 , respectively.

It is noted that at the cmc, although the fraction of "bound-Br" is more than $80 \%$ for DTAB, ${ }^{37}$ it is only around $60 \%$ for DeMIMB. If one considers that both DeMIMB and DTAB molecules have a rather bulky head group with a similar geometrical size as shown in Fig. 3 and similar surface excess concentration $\Gamma^{\mathrm{H}, \mathrm{cmc}}$ as shown in Fig. 2, the smaller value of $a_{2, \mathrm{cmc}}$ for DeMIMB suggests that the surfactant ions with "bound-Br" are more closely packed for DeMIMB than for DTAB. This may be because that imidazolium cations can take a kind of stacking alignment because of the plain structure of the imidazolium ring, but trimethylammonium cations can not do so because of the symmetrical structure.

The results from the XAFS analysis demonstrated above provide at least two more new insights with respect to the structure of adsorbed films of DeMIMB at the saturated adsorption. The first is that bromide ions are still solvated by around four water molecules, which is similar to the case of usual cationic surfactant, DTAB. ${ }^{37}$ The second is that XAFS spectrum of the adsorbed film could be expressed by the sum of two kinds of spectra $\chi_{1}$ and $\chi_{2}$. This indicates that the adsorbed films consist of two states, free and bound, and there is no intermediate situation for adsorbed surfactant molecules. Although the $\Gamma^{\mathrm{H}}$ value changes gradually and therefore it seems reasonable for adsorbed molecules to change gradually their conformation at the interface, the XAFS spectrum substantiates that the proportion of two states changes gradually with increasing the bulk concentration and this leads to the gradual increase in the surface excess concentration. The most conceivable situation is that the adsorbed molecules are definitely not homogeneously dispersed but that domains (islands or clusters) corresponding to $\chi_{2}$ are dispersed in the adsorbed films corresponding to $\chi_{1}$. A regular and rather tight stacking of imidazolium rings may be formed in the domains.

\section{Acknowledgements}

This work was supported by Grant-in-Aid for Scientific Research on Priority Area (No. 18445026) from the Ministry of Education, Science, Sports and Culture. This work has been performed under the approval of the Photon Factory Advisory Committee (Proposal No. 2006 G108), which was much appreciated.

\section{References}

1. J. S. Wilkes, J. A. Levisky, R. A. Wilson, and C. L. Hussey, Inorg. Chem., 1982, 21, 1263.

2. J. S. Wilkes and M. J. Zaworotko, J. Chem. Soc. Chem. Сотmun., 1992, 13, 965.

3. S. C. Fry and N. J. Pienta, J. Am. Chem. Soc., 1992, 107, 9366.

4. J. A. Boon, J. A. Levisky, J. L. Pflug, and J. S. Wilkes, J. Org. Chem., 1986, 51, 480.

5. M. J. Earle and K. R. Seddon, Pure Apple. Chem., 2000, 72, 1391.

6. J. Reiter, J. Vondrak, J. Michalek, and Z. Micka, Electrochim. Acta, 2006, 52, 1398.

7. B. M. Quinn, Z. Ding, R. Moulton, and A. J. Bard, Langmuir, 2002, 18, 1734.

8. H. Kamimura, T. Kubo, I. Minami, and S. Mori, Tribol. Int., 2007, 40, 620 .

9. B. Yu, F. Zhou, Z. Mu, Y. Liang, and W. Liu, Tribol. Int., 2006, 39, 879.

10. A. A. Fannin, J. D. A. Floreani, L. A. King, J. S. Landers, B. J. Piersma, D. J. Stech, R. L. Vaughn, J. S. Wilkes, and J L. Williams, J. Phys. Chem., 1984, 88, 2614.

11. J. D. Holbrey and K. R. Seddon, J. Chem. Soc., Dalton Trans., 1999, 13, 2133.

12. S. Tsuzuki, H. Tokuda, K. Hayamizu, and M. Watanabe, $J$. Phys. Chem. B, 2005, 109, 16474.

13. K. Kim, B. Shin, H. Lee, and F. Ziegler, Fluid Phase Equilib., 2004, 218, 215.

14. J. G. Huddleston, A. E. Visser, W. M. Reichert, H. D. Willauer, G. A. Broker, and R. D. Rogers, Green Chem. 2001, 3, 156

15. D. F. Evans and H. Wennerström, "The Colloidal Domain", 2nd ed., 1999, Wiley-VCH, New York, 130.

16. T. J. Gannon, G. Law, P. R. Watson, A. J. Carmichael, and K. R. Seddon, Langmuir, 1999, 15, 8429.

17. G. Law, P. R. Watson, A. J. Carmichael, and K. R. Seddon, Phys. Chem. Chem. Phys., 2001, 3, 2879.

18. G. Law and P. R. Watson, Chem. Phys. Lett., 2001, 345, 1.

19. T. Iimori, T. Iwahashi, H. Ishii, K. Seki, Y. Ouchi, R. Ozawa, H. Hamaguchi, and D. Kim, Chem. Phys. Lett., 
2004, 389, 321.

20. E. Slolutskin, B. M. Ocko, L. Taman, I. Kuzmenko, T. Gog, and M. Deutsch, J. Am. Chem. Soc., 2005, 127, 7796.

21. S. Rivera-Rubero and S. Baldelli, J. Phys. Chem. B, 2006, 110, 4756; C. S. Santos and S. Baldelli, J. Phys. Chem. B, 2007, 111, 4715.

22. M. Aratono, N. Toh, K. Shimamoto, T. Matsuda, H. Matsubara, and T. Takiue, unpublished.

23. T. Matsuda, Y. Mishima, A. Saeid, H. Matsubara, T. Takiue, and M. Aratono, Colloid Polym. Sci., 2007, 285, 1699.

24. T. Iimori, T. Iwahashi, K. Kanai, K. Seki. J. Sung, D. Kim, H. Hamaguchi, and Y. Ouchi, J. Phys. Chem. B, 2007, 111, 4860.

25. R. Ishimatsu, N. Nishi, and T. Kakiuchi, Langmuir, 2007, 23, 7608 .

26. "Kagaku Binran", ed. Chemical Society of Japan, 5th ed., 2004, Maruzen, Tokyo.

27. J. L. Lando and H. T. Oakley, J. Colloid Interface Sci., 1967, 25, 526.

28. I. Watanabe and H. Tanida, Anal. Sci., 1995, 11, 525 .

29. I. Watanabe, H. Tanida, S. Kawauchi, M. Harada, and M. Nomura, Rev. Instrum., 1997, 68, 3307.

30. T. Takiue, Y. Kawagoe, S. Muroi, R. Murakami, N. Ikeda, M. Aratono, H. Tanida, H. Sakane, M. Harada, and I. Watanabe, Langmuir, 2003, 19, 10803.

31. M. Aratono, K. Kashimoto, T. Matsuda, S. Muroi, Y. Takata, N. Ikeda, T. Takiue, H. Tanida, and I. Watanabe,
Langmuir, 2005, 21, 7398.

32. Y. Hayami, H. Ichikawa, A. Someya, M. Aratono, and K. Motomura, Colloid Polym. Sci., 1998, 276, 595.

33. H. Matsuki, N. Ando, M. Aratono, and K. Motomura, Bull. Chem. Soc. Jpn., 1989, 62, 2507.

34. K. Motomura, M. Aratono, N. Matubayasi, and R. Matuura, J. Colloid Interface Sci., 1978, 67, 247.

35. M. Harada, T. Okada, and I. Watanabe, J. Phys. Chem. B, 2003, 107, 2275 .

36. K. Kashimoto, Y. Takata, T. Matsuda, N. Ikeda, H. Matsubara, T. Takiue, M. Aratono, H. Tanida, and I. Watanabe, Langmuir, 2006, 22, 8403.

37. M. Aratono, K. Shimamoto, Y. Jimura, M. Hoshide, H. Tanida, T. Ozeki, H. Matsubara, T. Takiue, and I. Watanabe, to be submitted to Langmuir.

38. I. Watanabe, H. Tanida, and S. Kawauchi, J. Am. Chem. Soc., 1997, 119, 12018.

39. H. Tanida, H. Sakane, and I. Watanabe, J. Chem. Soc. Dalton Trans., 1994, 15, 2321.

40. A. L. Ankudinov, B. Ravel, J. J. Rehr, and S. D. Conradson, Phys. Rev. B, 1998, 58, 7565; J. J. Rehr and R. C. Albers, Rev. Mod. Phys., 2000, 72, 621.

41. Y. Sawa, T. Miyanaga, H. Tanida, and I. Watanabe, J. Chem. Soc. Faraday Trans., 1995, 91, 4389.

42. H. Ohtaki and T. Radnai, Chem. Rev., 1993, 93, 1157.

43. N. Otomo, K. Arakawa, M. Takeuchi, T. Yamaguchi, and H. Otaki, Bull. Chem. Soc. Jpn., 1981, 54, 1314. 